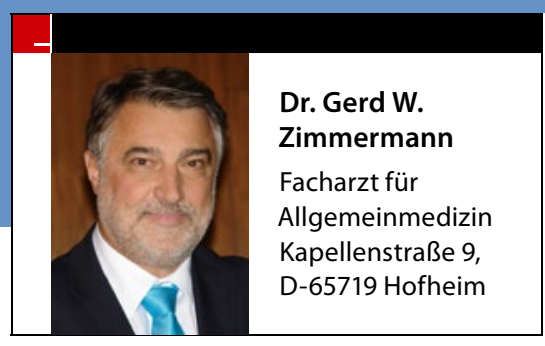

- Der Bewertungsausschuss von Kassen und KBV beschließt in unregelmäßigen Abständen Kommentierungen bzw. Auslegungen einzelner EBM-Ziffern. Diese haben eine hohe Verbindlichkeit und werden deshalb zum Bestandteil des EBM. Ab dem 1. Oktober 2015 gibt es nun zwei neue Interpretationsbeschlüsse.

Der erste betrifft die Besuchsziffern: Wird ein Arzt zu einem dringenden Besuch gerufen, trifft den Patienten aber nicht (mehr) an, darf er trotzdem die EBMNr. 01411 bzw. 01412 berechnen. Die Dringlichkeit des Besuchs und auch die Abwesenheit sollte dabei in der Patientenakte dokumentiert werden.
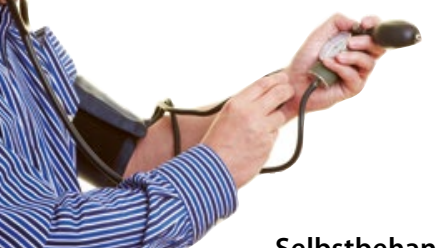

Selbstbehandlung bringt nur noch den Verwaltungskomplex.

\title{
Abrechnung von Besuchen und Selbstbehandlung eingeschränkt
}

Ein weiterer Interpretationsbeschluss beschäftigt sich mit einer Besonderheit in der GKV: „Bei Selbstbehandlung kann ein Vertragsarzt die Gebührenordnungsposition 01430 berechnen. Daneben sind Leistungen des Abschnitts II und Gesprächsleistungen nicht berechnungsfähig."

Nebenbei bestätigt dieser Beschluss, dass Vertragsärzte in voller Höhe Beiträge zur GKV zahlen dürfen. Wenn sie selbst krank werden, müssen sie der Kasse aber einen Arztrabatt geben, wenn sie sich (kostengünstig) selbst behandeln.

\section{MMW-Kommentar}

Beide Beschlüsse sind streng genommen überflüssig, denn die Regeln gibt es längst sie wurden bisher lediglich nicht im EBM fixiert. Für sogenannte unvollendete Hausbesuche gab es bereits eine gesicherte Rechtsprechung, die feststellte, dass auch dann die Besuchsgebühr und die Kilometerpauschale angesetzt werden können, wenn der Patient nicht angetroffen wird. Der Beschluss unterstreicht dies nur noch einmal explizit - ist aber in anderer Hinsicht ein Rückschritt. Er legt jetzt nämlich fest, dass der Arzt nur bei dringenden Hausbesuchen nach den Nrn. 01411 bzw. 01412 so verfahren kann. Gleiches gilt für die Regelung beim Ansatz der Nr. 01430. Dieser ist bei Selbstbehandlung nun Pflicht. Eigentlich kein Problem, zumal es im Beschluss des Bewertungsausschusses noch heißt, dass daneben nur Gesprächsleistungen und andere Leistungen des Abschnitts II ausgeschlossen sind. So erscheint sogar eine Berechnung der Versichertenpauschale möglich. Allerdings versteckt sich in der Legende der Nr. 01430 ein Haken: Neben ihr dürfen im gesamten Quartal keine anderen Leistungen berechnet werden! Der Pflicht-Ansatz schließt also automatisch alle anderen Ziffern aus. Lediglich ein Mehrfachansatz der Nr. 01430 ist möglich.

Beachtenswert ist dabei, dass sich diese Regelung nur auf den Arztfall bezieht. Lässt man sich also etwa vom Praxispartner behandeln, kann dieser seine Leistungen - versehen mit seiner LANR - uneingeschränkt berechnen.

\section{Mehr als 2.000 MVZ in Deutschland - größte Medizinergruppe sind die Hausärzte}

_ Laut den aktuellen Zahlen (Stand: 31. Dezember 2014) gibt es in Deutschland 2.073 Medizinische Versorgungszentren (MVZ) mit 13.465 Ärztinnen und Ärzten. Darunter sind 1.346 Vertragsärzte, von denen $89 \%$ mit einem ganzen Versorgungsauftrag arbeiten. 12.119 MVZ-Ärzte sind im Angestelltenverhältnis tätig, 58\% von ihnen in Teilzeit. 38,4\% der MVZ-Träger sind Krankenhäuser. Die meisten Krankenhaus-MVZ in absoluten Zah- len gibt es in Bayern, Niedersachsen und Baden-Württemberg. In Hamburg und Thüringen ist der Anteil der MVZ-Ärzte gemessen an den regional zugelassenen Vertragsärzten am größten. Einem MVZ stehen im Bundesdurchschnitt ca. 49 Einzel- bzw. Gemeinschaftspraxen gegenüber. Während in Hamburg ein MVZ auf 20.790 Einwohner kommt, gibt es in Baden-Württemberg ein MVZ pro 71.351 Einwohner.

\section{MMW-Kommenta}

MVZ gründen sich sowohl in städtischen als auch ländlichen Gebieten, meist allerdings in Kernstädten sowie Ober- und Mittelzentren. Durchschnittlich arbeiten 6,5 Ärzte in einem MVZ. Mit 1.913 stellen die Hausärzte den größten Anteil des ärztlichen Personals, gefolgt von Internisten (1.576) und Chirurgen (1.021). In der Rechtsform überwiegt die GmbH mit 1.278, 512 Zentren sind GbR. 